\title{
Application of Brillouin-Based Continuously Tunable Optical Delay Line to Contention Resolution Between Asynchronous Optical Packets
}

\author{
Claudio Porzi, Sanghoon Chin, Andrea Trita, Francesco Fresi, Gianluca Berrettini, Gabor Mezosi, Paolo Ghelfi, \\ Guido Giuliani, Luca Potì, Marc Sorel, Luc Thévenaz, and Antonella Bogoni
}

\begin{abstract}
A novel approach for resolution of contentions between asynchronous optical packets, exploiting a self-synchronizing mechanism by means of dynamic all-optical tunable delay line based on stimulated Brillouin scattering in fibers, is reported. Time delay between contending optical packets is mapped into the output timing of a contention-detecting pulse produced by the tunable delay line. The output pulse from the delay line triggers an integrated all-optical flip-flop that generates a synchronized gating signal driving a semiconductor-based all-optical switch, for low-priority packets deflection. Correct operation of the proposed scheme without bit loss or packet fragmentation is demonstrated, and error-free operation for the switched packets is reported under different cases of relative delay between incoming optical packets. The scheme operates entirely in the photonic domain and exploits integrated optical switches and a passive optical-delay line for minimized node latency. Extension to symmetric operation allowing priority swapping between the input packets is also proposed.
\end{abstract}

Index Terms - All-optical variable delay lines, contention resolution, asynchronous packet switched networks, stimulated Brillouin scattering, all-optical flip-flops, integrated optical gates.

\section{INTRODUCTION}

\section{A} LL-OPTICAL TUNABLE DELAY LINES (AOTDLs) are expected to play an important role in future fiber-optic telecommunications and information processing systems. By performing operations like data synchronization, optical buffering, pattern recognition, and writing/reading of random access memories at a photonic level, a greater flexibility and a reduced latency time in optical data routers and processing systems can be achieved. Over the last few years, several different types of all-optical tunable delay lines have been proposed

Manuscript received February 08, 2013; revised July 15, 2013; accepted July 15, 2013. Date of publication July 30, 2013; date of current version August 14, 2013. This work was supported in part by the European Community's Seventh Framework Program under grant agreement no. 219299 (GOSPEL project).

C. Porzi, F. Fresi, and G. Berrettini are with TeCIP, Scuola Superiore Sant'Anna, 56124 Pisa, Italy (e-mail: claudio.porzi@sssup.it).

S. Chin, and L. Thévanaz are with Institute of Electrical Engineering, Ecole Polytechnique Federale de Lausanne, Lausanne, Switzerland. (e-mail: sanghoon.chin@epfl.ch; luc.thevenaz@epfl.ch).

A. Trita is with IMEC, Department of Information Technology, Ghent University, 9000 Gent, Belgium.

G. Giuliani is with Optoelectronics Group, Dipartimento di Elettronica, Università di Pavia, i-27100 Pavia, Italy.

M. Sorel and G. Mezosi are with the Department of Electronics and Electrical

Engineering, University of Glasgow, Glasgow G12 8LT, UK.

P. Ghelfi, L.Poti, and A. Bogoni are with CNIT, 56124 Pisa, Italy.

Digital Object Identifier 10.1109/JLT.2013.2275253
[1]-[12]. The most straightforward method to implement an AOTDL is by means of several fixed-delay fiber spans connected by a series of space switches [1], [2]. In this type of delay line, however, a fine tuning of signal delaying is essentially limited, and electrical control signals are required. Thus, for the generation of continuous signal delaying, several techniques operating entirely at a photonic level have also been proposed, using a variety of approaches such as different slow-light mechanisms [3]-[8], wavelength conversion accompanying a dispersive medium [9]-[11] or time-frequency conversion [12]. Most techniques, however, suffer from inherent limitations in terms of signal bandwidth and/or maximum achievable time delay without incurring in significant signal distortion. For these reasons, no practical system demonstration of AOTDLs has been implemented up to date.

Recently, a novel type of all-optical delay line based on dynamic Brillouin grating (DBG) has been proposed and implemented in polarization maintaining (PM) fiber [13]. In this type of delay line, grating reflectors can be localized at a position in the PM fiber and the grating position can be dynamically reconfigured at any preset position from an end of the PMF to the other end of the PMF. As a result, the maximal achievable time delay is essentially determined by the physical length of the PMF, so dynamic signal delaying up to the order of few microseconds with a minor signal distortion can be readily obtained. This technique shows a potential for implementing practical and effective AOTDL, suitable to realize synchronization stages for optical packet switched networks. This feature allowed us to exploit the DBG-based AOTDL in a practical system application such as all-optical contention-resolution between asynchronous packets for optical packet-switched networks [14]. Contention at a network node occurs when two or more packets at the same wavelength compete for the same switch output port within an overlapping interval of time. Several all-optical approaches for solving contentions have been investigated in the past years [15]-[20]. Contention between packets can be solved in the optical domain by exploiting optical buffering, or wavelength conversion, or space deflection [21]. One of the most challenging issues in OPSN is to handle contention between packets asynchronously arriving at a network node [22]. In all cases, efficient operation can be achieved by properly synchronizing the packets at the switching elements. To achieve this task, because of the lack of practical random access memories in the optical domain, a typical synchronization stage in OPSN is comprised of a series of switches and delay lines [23]-[27]. In these 
approaches, the total delay is changed by properly setting the switches through an electrical control pane. By avoiding the use of the electrical control plane, the overall latency of the node can be reduced. Real-time all-optical approaches for contention resolution between asynchronous packets, avoiding the use of fixed delay-line buffering and electrical control plane, have been also reported in the past years [19], [20], where a latching mechanism is exploited so that if a packet requests for a node output which is currently busy, it is automatically forwarded to a different lightwave path.

Here, we describe our proposed novel all-optical asynchronous contention resolution approach, exploiting a DBG-based continuously tunable AOTDL, enabling self synchronization of the switching signals within the routing node. The scheme is suitable for fixed-size packets. The results reported in [14] are reviewed in more details and an extension of the original scheme enabling improved network flexibility is included. We demonstrate the effectiveness of the scheme in a contention resolution experiment between asynchronous packets encompassing OOK-modulated data at $10 \mathrm{~Gb} / \mathrm{s}$ and an optical header section comprised of a single synchronization pulse. Due to its operation principle, the scheme is transparent to the modulation format and rate of the data in the packets, thus allowing operation at higher data rate and with different modulation formats. For this experimental demonstration, packet priority is determined by the input port of the packet in the switching node; a more general architecture enabling priority selection on the basis of information contained in priority labels, regardless of the position of the packets at the node input, is also described.

The paper is organized as follows. In Section II, we introduce the operation principle of the self-synchronization stage based on an AOTDL exploiting SBS in PM fiber. Section III describes the experimental implementation of the setup for contention resolution between asynchronous packets; the corresponding results are illustrated in Section IV. In Section V, we propose and discuss the extension of the operation to the case in which priority can be swapped between packets accordingly to the value of the label in their priority field. Finally, the concluding discussions are presented in Section VI.

\section{PRINCIPLE OF OPERATION}

The proposed architecture for asynchronous contention resolution operation is illustrated in Fig. 1. Two packets, Packet $\mathrm{A}$ and Packet B at an identical wavelength $\lambda_{\mathrm{pck}}$ and duration $\mathrm{T}_{\mathrm{pck}}$, enter the node inputs IN 1 and IN 2, respectively. The two packets enter into the node with a random time delay, but have the same intended output port, which is indicated as node out in the figure; we first assume that packets entering from IN 1 port are the high-priority packets.

In our architecture, a synchronization pulse (SP) is temporally placed in front of each packet, and is used for signaling the arrival timing of the corresponding packets. When the packets and SPs enter the routing node, the SPs are selected to be circulated to a delay evaluation stage (DES). On the other hand, Packet A, which enters the high-priority input port IN 1, is delivered to the intended node output, node out, whereas Packet B,

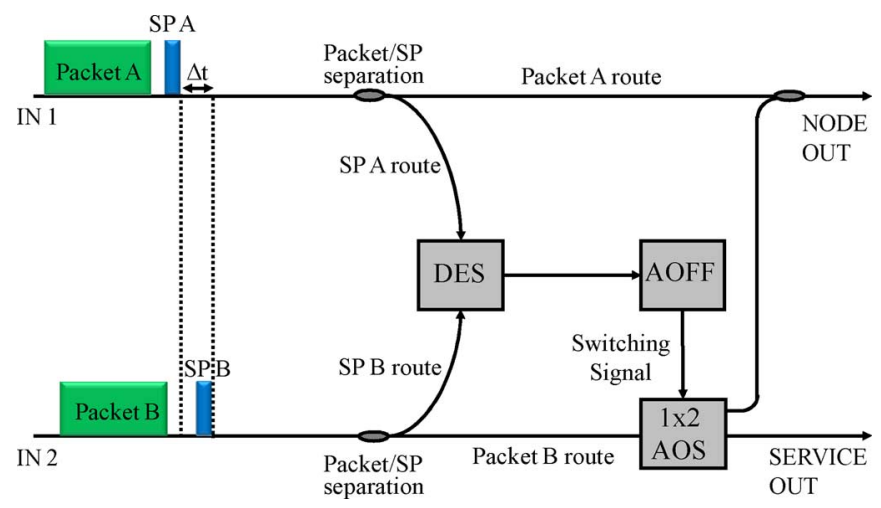

Fig. 1. Operation principle of the self-synchronizing scheme for asynchronous contention resolution; (SP: synchronization pulse, DES: delay evaluation stage, AOFF: all-optical flip-flop, AOS: all-optical switch).

entering the low-priority input port IN 2 , is delivered to a $1 \times 2$ all-optical switch (AOS). The role of the AOS is to deflect the low-priority packet to node out or to an alternative route (service out) depending on its $O F F$ - and $O N$-states, respectively. The status of the AOS can be altered by an all-optical flip-flop (AOFF), which is triggered by the output signal produced by the delay-evaluation stage (DES). The role of the DES, which is the core element of the self-synchronizing node, is to detect occurrence of contention by generating a contention-detecting pulse at its output, being the output timing of the contention-detecting pulse a function of the relative time delay $\Delta t$ between the two SPs (i.e., the relative delay between Packet A and Packet B). The pulse leaving the DES is then delivered to the AOFF, operating in a set-reset mode, in order to produce a gating signal which has the same duration of the packets and drives the $1 \times 2 \mathrm{AOS}$. In the absence of contention, no contention-detecting pulse is generated by the DES, and no switching signal is produced by the AOFF. As a result, Packet B is deflected toward the intended node output, node out, through the $1 \times 2$ AOS in the $O F F$-state. On the contrary, in case of contention, a triggering pulse is delivered from the DES to the AOFF after a proper time delay which is determined by the relative delay between two contending packets, $\Delta \mathrm{t}$. The switching signal generated by the $\mathrm{AOFF}$, which is responsible for commuting the $1 \times 2$ AOS in the $O N$-state, is thus automatically synchronized with Packet B at the switch input, allowing deflection of the low-priority Packet B without packet fragmentation toward the service out port, in case of contention. Depending on the adopted contention-resolution strategy, the deflected packets at the service out port could be either routed onto a different lightwave path or be re-input to the node after a proper delay or sent to a wavelength-conversion stage and directed at the intended node output at a different wavelength. Based on network topology, a new SP pulse might be required for the output packets. This can be implemented by tapping a fraction of the SPs at the DES input and recombining it with the output packets by means of passive couplers/splitters and fixed delay lines.

Fig. 2 depicts the details of the implementation of the DES, which is comprised of an all-optical wavelength conversion (WC) stage, a frequency shifter (FS) and the continuously tunable, optically-controllable, AOTDL based on stimulated 


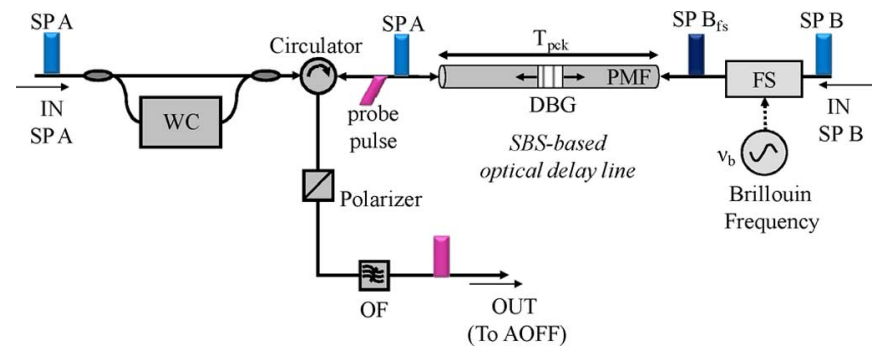

Fig. 2. Implementation of the DES with the continuously tunable BDG-based AOTDL (WC: wavelength converter, OF: optical filter; SBS: stimulated Brillouin Scattering; PMF: polarization maintaining fiber, FS: frequency shifter; DBG: dynamic Brillouin grating; AOFF: all-optical flip-flop).

Brillouin scattering (SBS) effect [13]. A span of polarization-maintaining (PM) fiber is used in the AOTDL and the transit time through the fiber is equal to the duration of the packets, $T_{\text {pck }}$.

A key to generate a self-synchronized switching signal feeding the $1 \times 2$ AOS relies on the generation and localization of dynamic Brillouin grating (DBG) in the PM fiber. When the two counter-propagating SP pulses are spectrally separated by the Brillouin frequency shift of the fiber, $\nu_{\mathrm{B}}$, an acoustic wave is created at a specific position in the fiber, where the two pulses have encountered. Then, the acoustic wave acts as a grating reflector under Bragg condition. In particular, in the situation where the two SP pulses are linearly polarized along a primary axis in PMF, the acoustic grating resonance appears at two distinct optical frequencies for light propagating along each primary axis, due to the high birefringence of the fiber. If the optical frequency of the SP pulse (the higher frequency among the two pulses) is matching the grating resonance in one primary axis, the same resonance will reflect light in the other primary axis at a frequency $\sim 45 \mathrm{GHz}$ above the pulse frequency in standard PMF [28].

In our DES system, the spatial location of the DBG along the PM fiber is basically determined by the difference between the input timing of the two SP pulses. A probe pulse that enters into the AOTDL following the SP A is then partially reflected from the BDG and is circulated to the optical Flip-Flop with a time delay which is a function of the grating position in the PM fiber. The reflected pulse is then used for the generation of a switching signal from the AOFF. Self-synchronization of the switching signals in the contention resolution node of Fig. 1 can then be enabled as explained in the following. As shown in Fig. 2, when the SP A pulse enters the DES linearly polarized along slow axis of the PM fiber, it is split into two branches; a fraction of the SP A pulse passes through the WC stage, for the generation of a probe pulse. The polarization of the probe pulses leaving the WC stage is aligned along the fast axis of the PMF, and is optical frequency is set to match the DBG frequency in the PM fiber. The probe pulse at the output of the WC stage is recombined, with an appropriate time delay, with the SP A and the two pulses are sent to the AOTDL from one end of the PM fiber span. In addition, the optical frequency of SP B is also shifted to $\nu_{\mathrm{B}}$ below the SP A frequency in the FS stage, for the efficient SBS interaction. The frequency shifted SP B pulse, hereafter named $\mathrm{SP}_{\mathrm{fs}}$, is also linearly polarized along slow

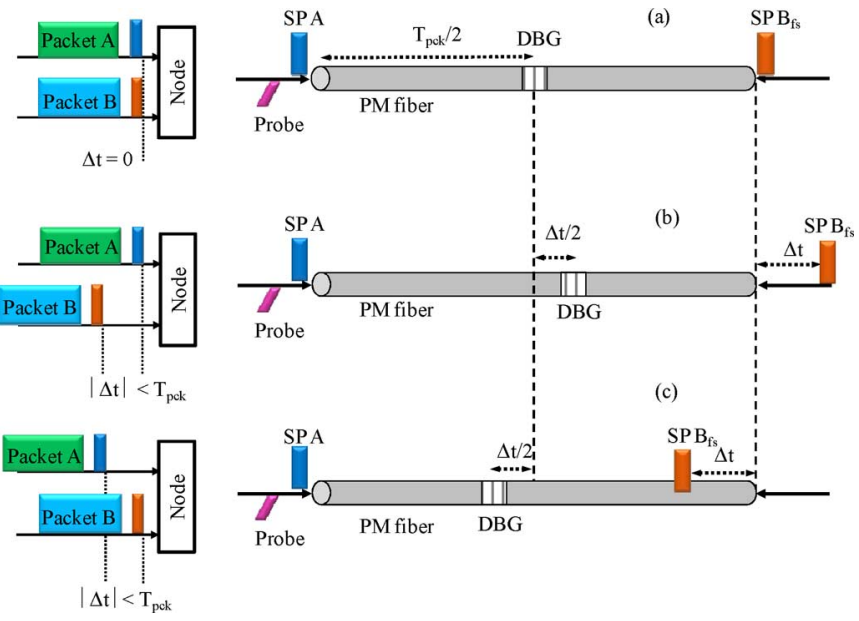

Fig. 3. Mechanism for self-synchronization with the BDG-based AOTDL.

axis of PM fiber and is sent to the AOTDL entering the opposite end of the PM fiber with respect to SP A. A dynamic grating is thus generated at the position where the two pulses encounter along the fiber. The probe pulse is then back-reflected from this point and sent to the AOFF trough a circulator. A polarizer and an optical filter (OF) are inserted after the circulator to filter out unwanted $\mathrm{SP}_{\mathrm{fs}}$ and residual back-reflected $\mathrm{SP} \mathrm{A}$, so that only the contention-detecting probe pulse is input to the AOFF. The operation of the DES is then described in the following. For the case of no contention, the two packets are completely separated in time at the node inputs. Thus, SP A and SP $\mathrm{B}_{\mathrm{fs}}$ do not encounter within the PM fiber, since its travel time is equal to the packets duration. Consequently, a DBG is not created in the fiber, and no contention-detecting signal is generated at the DES output, the AOFF remains idle and no switching signal is sent to the $1 \times 2$ AOS from the AOFF output. As a result, the $1 \times 2 \mathrm{AOS}$ is in the $O F F$-state, and both Packet B and Packet A are routed toward the intended node output (node out in Fig. 1), for this no-contention case.

For the case in which contention occurs, let us first assume that the two packets arrive synchronously at the node inputs. SP A and SP $B_{\mathrm{fs}}$ enter thus the PM fiber at the same instant, as shown in Fig. 3(a). A DBG is created in the middle of the fiber, and a contention-detecting pulse (i.e., the reflected probe) is produced at the DES output with an additional delay introduced by the AOTDL of $\mathrm{T}_{\mathrm{pck}}$. A triggering set/reset signal is then delivered to the AOFF, and a switching signal slightly larger than $\mathrm{T}_{\mathrm{pck}}$ is generated at the AOFF output. By properly fixing the path of Packet $\mathrm{B}$ in the node, the switching signal at the $1 \times 2$ AOS can be made synchronous with Packet B for this case of same arrival instant. The switching signal from the AOFF commutes the $1 \times 2 \mathrm{AOS}$ in the $O N$-state, and Packet B is then deflected toward the output named service out in Fig. 1 for this contention case. If Packet $\mathrm{B}$ enters the node delayed (advanced) with respect to Packet A by $\Delta \mathrm{t}$, being $0<\Delta \mathrm{t}<$ $\mathrm{T}_{\mathrm{pck}}\left(-\mathrm{T}_{\mathrm{pck}}<\Delta \mathrm{t}<0\right)$, then SP A enters the fiber before (after) SP $\mathrm{B}_{\mathrm{fs}}$ by the same amount of time (see Fig. 3(b) and (c), respectively). The DBG is then created farther from (closer to) the probe input side, with a time offset of $\Delta t / 2$ with respect to the middle of the PM fiber span. The reflected probe pulse 


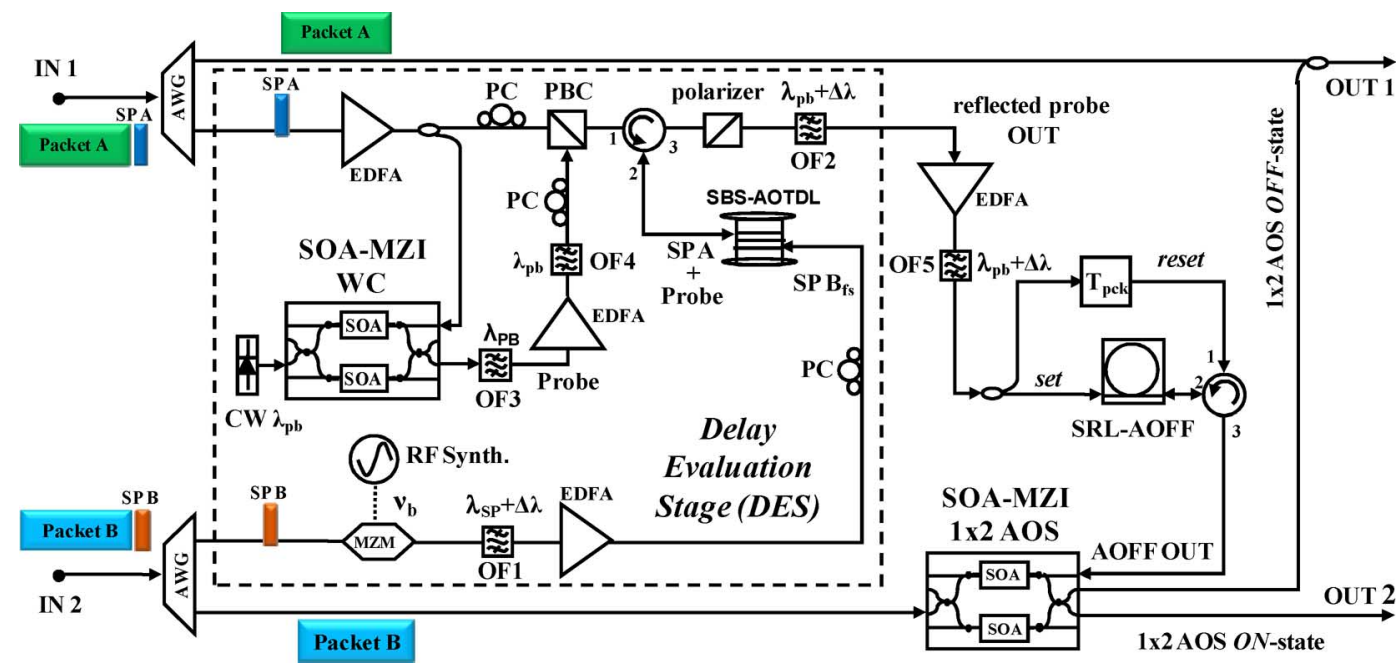

Fig. 4. Experimental setup (AWG: arrayed waveguide grating; EDFA: arbium-doped fiber amplifier; PC: polarization controller; PBC: polarization beam coupler; OF: optical filter; SRL-AOFF: semiconductor ring laser all-optical flip-flop, SOA-MZI: semiconductor optical amplifier Mach-Zehnder interferometer, WC: wavelength converter, MZM: Mach-Zehnder modulator).

detecting the contention is correspondingly delayed (advanced) by $\Delta \mathrm{t}$, and the corresponding switching signal generated by the AOFF is still synchronized with Packet $\mathrm{B}$ at the $1 \times 2$ AOS, thus correctly deflecting Packet B toward service out.

Extension to multiple input ports can be obtained by conveniently replicating the DES in order to detect any possible contention between the incoming packets. In particular, each SP must detect a possible contention with all the SPs coming from lower priority ports. The contention-detecting pulses generated by the DESs can then conveniently drive AOFFs and AOSs to deflect the contending packets toward different service outputs. In this way, for $\mathrm{N}$ input ports, the number of AOFFs, DESs and $1 \times 2$ AOSs would thus be given by $\sum_{x=1}^{N-1}(N-x)$.

\section{EXPERIMENTAL IMPLEMENTATION}

The experimental set-up we adopted for demonstrating asynchronous contention resolution is shown in Fig. 4. Two optical packets at the wavelength $\lambda_{\text {pck }}$ of $1551.7 \mathrm{~nm}$, comprising OOK-modulated data at $10 \mathrm{~Gb} / \mathrm{s}$ are generated by means of a pattern generator (PG) and high speed electro-optic MachZehnder modulators (MZMs). Each packet comprises an out-ofband header section embedding 5 ns-long SPs at $\lambda_{\mathrm{SP}}=1557.8$ $\mathrm{nm}$. Packets' duration was chosen to be $\mathrm{T}_{\mathrm{pck}}=500 \mathrm{~ns}$ (corresponding to $\sim 100 \mathrm{~m}$ of PM fiber for the SBS-based AOTDL). As depicted in the figure, Packet A and SP A enter the circuit from IN 1, which is the high-priority port for this experiment, whereas Packet B and SP B enter IN 2 port of the node, the low-priority input port of the node. In the next Section, we will describe how to extend the operation to allow for priority swapping between the input ports by using extra label pulses containing the information on the packet priority. As shown in Fig. 4, data packets and SPs are separated using arrayed waveguide grating (AWG) optical filters. Packet A is sent to the intended node output, OUT 1 while the data of Packet B are sent to a $1 \times 2 \mathrm{AOS}$, which is implemented with semiconductor optical amplifiers in Mach-Zehnder interferometer configuration (SOA-MZI). The SPs are delivered to the delay DES, comprising a MZM-based frequency shifter, a SOA-MZI wavelength converter (WC) and a span of PM fiber implementing the BDG-based AOTDL. Erbium-doped fiber amplifiers (EDFAs) are also used to boost the optical powers of the signals along the different stages of the circuit.

The MZM producing the frequency-shifted replica of SP B, $\mathrm{SP} \mathrm{B}_{\mathrm{fs}}$, is driven by a sinusoidal tone matching the Brillouin frequency of the PM fiber in the BDG-based AOTDL $\left(\nu_{\mathrm{b}} \sim 10.53\right.$ $\mathrm{GHz}$ ). A bandpass optical filter (OF 1), detuned by $\Delta \lambda$ with respect to $\lambda_{\mathrm{SP}}$, is used to select the optical frequency spaced below that of SP B (and SP A) by $\nu_{\mathrm{b}}$. At the AWG output, SP A is amplified by an EDFA and split into two paths. One path is sent to a WC stage to generate the probe signal, whereas the second path is connected to one input port of a polarization beam coupler (PBC) with polarization-maintaining (PM) input/output fibers. A polarization controller (PC) is used to maximize the transmission of SP A through the PBC. The output port of the PBC is connected to port 1 of a PM optical circulator, CIRC 1. SP A exits thus from port 2 of CIRC 1 to enter the PM fiber in the SBS-based AOTDL, linearly polarized along its fast axis. Similarly, the polarization state of SP $B_{\mathrm{fs}}$ leaving OF 1 (after being amplified by an EDFA) is adjusted with a PC so that SP $B_{f s}$ is also aligned along the PM fiber fast axis. Thus, both SP $\mathrm{A}$ and $\mathrm{SP} \mathrm{B}_{\mathrm{fs}}$ are launched from opposite ends of the PM fiber linearly polarized along its fast axis. As depicted in Fig. 4, a fraction of SP A is also sent to the WC stage, acting as a pump signal in the SOA-MZI. In particular, SP A enters only a single SOA of the structure whereas a probe signal provided by a $\mathrm{CW}$ laser at $\lambda_{\mathrm{pb}}$ is applied to the input port which is common to both SOAs. The presence of SPA depletes the carrier density in the semiconductor amplifier, causing an additional phase shift only for the probe light that travels in the upper branch of the interferometer. The SOA-MZI has been properly biased such that the probe signal at $\lambda_{\mathrm{pb}}$ experiences almost complete destructive interference at the output port in absence of the pump SP A. When a pump SP A enters the WC stage, the extra phase shift leads to constructive interference at the output port and 
the transmission of the probe light is correspondingly switched to the $O N$ state. A probe pulse at $\lambda_{\mathrm{pb}}$ is then produced at the SOA-MZI output each time an SP A pulse enter the WC stage. The wavelength of the $\mathrm{CW}$ laser, $\lambda_{\mathrm{pb}}$, corresponds to an optical frequency which is $\sim 45 \mathrm{GHz}$ below that of SP A, $\lambda_{\mathrm{SP}}$. In particular, $\lambda_{\mathrm{pb}}$ is chosen to matches the Bragg wavelength of the DBG generated in the SBS-ODL by SP A and SP $\mathrm{B}_{\mathrm{fs}}$ for a signal propagating along the slow axis of the PM fiber. At the output of the SOA-MZI WC, the probe pulse is selected by an OF centred at $\lambda_{\mathrm{pb}}$, and subsequently amplified by an EDFA and filtered again. The probe pulse then enters the second input port of the $\mathrm{PBC}$ so that, at its output, its polarization state is orthogonal to that of SP A (i.e., probe pulse aligned on the slow axis of the PM fiber). A PC is used to maximize transmission of the probe through the PBC. After PBC and circulator, the probe pulse enters SBS-based AOTDL following SP A with a delay of $\sim 2.5$ ns.

When a DBG is created by the interaction of SP A and SP $\mathrm{B}_{\mathrm{fs}}$, the reflected probe pulse is retrieved through port 3 of the optical circulator. The probe signal is isolated by a polarizer (POL) aligned with the probe polarization state, and filtered by an optical bandpass filter OF 2 (bandwidth: $0.15 \mathrm{~nm}$ ). This ensures strong rejection of the orthogonally-polarized $\mathrm{SP} \mathrm{B}_{\mathrm{fs}}$ that co-propagates with the reflected probe and back-reflected light from SP A. Since the DBG is moving at the velocity of an acoustic wave inside the PM fiber in the SBS-based AOTDL, the reflected probe signal experiences an ordinary Brillouin shift of $\nu_{\mathrm{b}}$ by the Doppler effect. At the output of the DES, the probe pulse is amplified and filtered with OF 5 , and subsequently split into two paths in order to create two delayed replicas which are sent to the set and reset ports of an all-optical flip-flop (AOFF). The delay between the set and reset pulse is slightly longer than $\mathrm{T}_{\text {pck }}$. The AOFF is based on a monolithic semiconductor ring laser (SRL) fabricated in InGaAs/InGaAlAs/InP MQW material [29]. The device is composed of a racetrack cavity coupled to two bus waveguides, which act as input/output ports for the device. AOFF operation is achieved thanks to the inherent directional bistability among the two counterpropagating directional modes supported by the cavity. The lasing direction of the SRL, which represents the state of the AOFF, can be addressed all-optically by means of optical pulses injected from the opposite sides of the bus waveguide, which force the lasing mode in the ring cavity to be either co- or counter-clockwise. Depending on the directional lasing operation, optical power is thus present only at either one end or the other of the bus waveguide. With reference to Fig. 4, the AOFF is initialized such that the lasing direction is clockwise and no signal is present at port 3 of the optical circulator connected to the $1 \times 2$ AOS. When a control pulse enters the AOFF from the left-hand side (set signal), the lasing direction is switched to counter-clockwise and a rising step-like optical signal is supplied to port 3 of the circulator. The same pulse, properly delayed, act as reset pulse when injected from the right-hand side (reset signal), switching back the AOFF to the counter-clockwise lasing mode. A rectangular optical pulse of duration slightly longer than $\mathrm{T}_{\mathrm{pck}}$ is then produced, which in turn controls the $1 \times 2 \mathrm{AOS}$ and synchronously deflects Packet B toward OUT 2 of the node in case of contention.

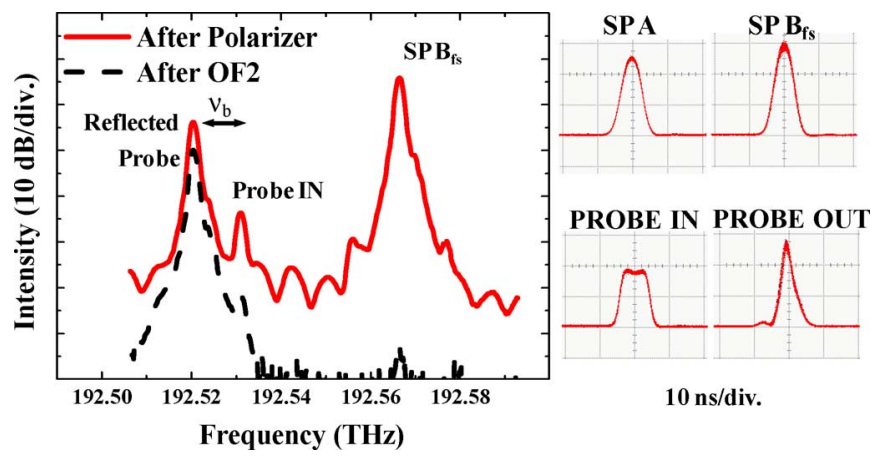

Fig. 5. Optical spectra at the output of the SBS-based ODL (left) and temporal traces of the SBS-based AOTDL input/output signals (right).

\section{RESULTS}

The SBS-based AOTDL output spectra for the contention case are shown in Fig. 5, after the polarizer (solid line) and at OF 2 output (DES output, dashed line). A strong reflected probe component (with the ordinary Doppler shift of $\nu_{\mathrm{b}}$ ) is evident. The oscilloscope traces of the signals at the input of the SBS-based AOTDL and the output reflected probe (after OF 5) for a case of contention are also shown in Fig. 5. The traces corresponds to the signals after EDFAs and OFs. The flat-top feature of the wavelength-converted probe in Fig. 5 is due to the cosine-squared shaped transmission characteristic of SOA-MZI. The oscilloscope traces of the packets, synchronizing signals, and AOFF output signal, for different contention/no contention cases are illustrated in Fig. 6. As can be seen from the figure, a contention-detecting pulse synchronous with the leading edge of the contending Packet $\mathrm{B}$, is generated for both the cases of Packet B partially delayed (cases of PCK A3-PCK B1 contention) or partially advanced with respect to Packet A (case of PCK A1-PCK B3 contention). No contention detecting pulse is generated in absence of contention (i.e., no time overlapping between input Packet A and Packet B). As a consequence, a switching signal is produced by the AOFF only for the cases of contention.

Since the AOFF output signal is synchronous with the contending Packet B, as illustrated in Fig. 6, space deflection of the input Packets $\mathrm{B}$ is correctly achieved for all the contention cases, as reported by the traces for the $1 \times 2 \mathrm{AOS} O F F$ - and $O N$-state outputs. By recollecting the $1 \times 2 \mathrm{AOS} O N$-state output with the high-priority packets path, Packets B are routed toward the intended node output OUT 1 when no contention occurs.

For the temporal traces of Fig. 6, the corresponding average power levels at the input of SBS-based AOTDL of SP A, SP B $\mathrm{B}_{\mathrm{fs}}$ and probe signal are $11 \mathrm{dBm}, 10 \mathrm{dBm}$, and $8 \mathrm{dBm}$, respectively. In the SOA-MZI WC, the CW input probe light is $\sim 0 \mathrm{dBm}$, whereas the average power level of the pump SP A signal is $\sim-17 \mathrm{dBm}$. The average power level of both the set and reset signals at the SRL-AOFF is $-7 \mathrm{dBm}$. Finally, the average power of the switching signal at the SOA-MZI $1 \times 2$ AOS is -12.5 $\mathrm{dBm}$ whereas the probe Packets B power is set to $-8 \mathrm{dBm}$. Variations of few $\mathrm{dB}$ around this typical levels can be fairly tolerated by the systems without significant degradation of its operation. 


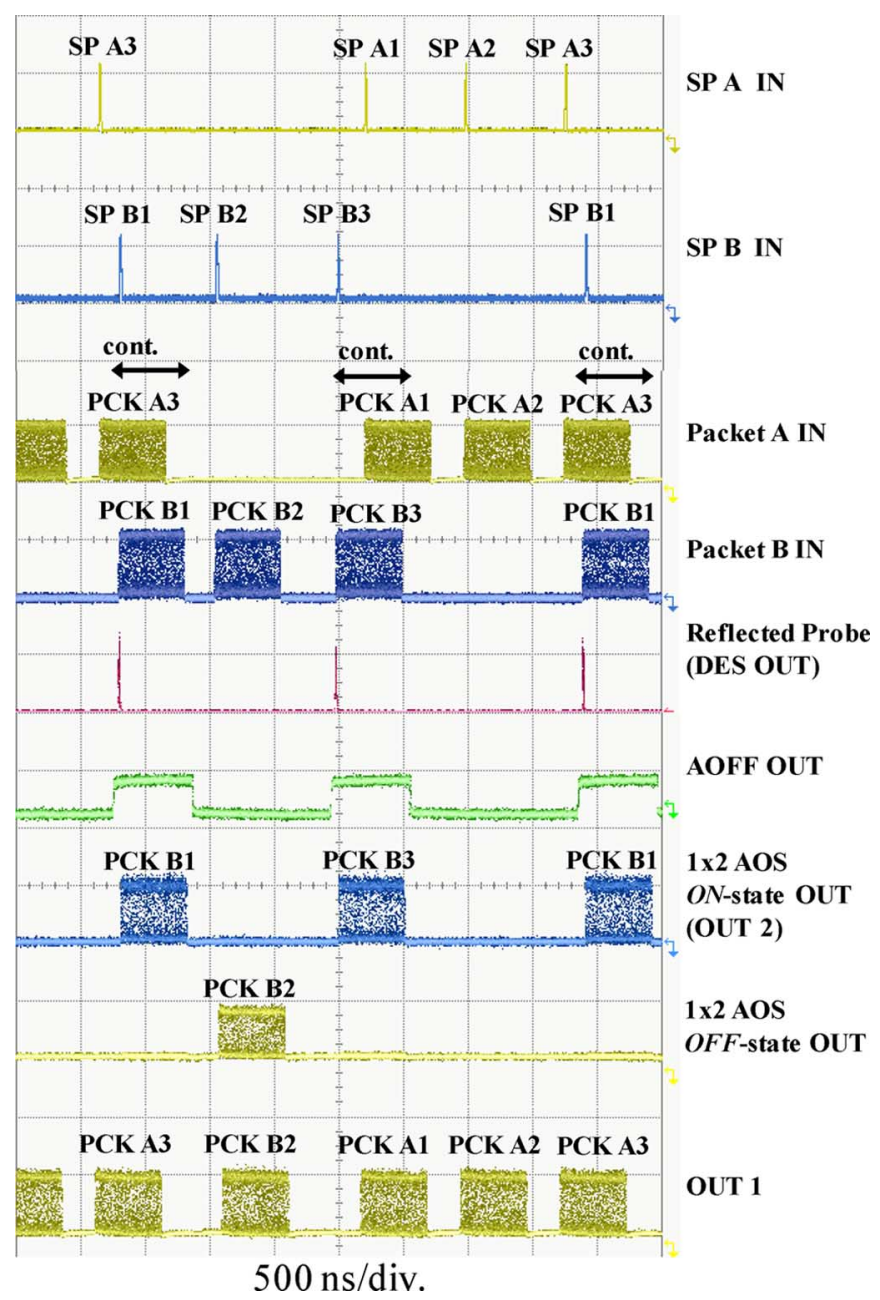

Fig. 6. Input/output oscilloscope traces of signals along the circuit of Fig. 4 for different contention/no contention cases; from top to bottom: input SP A, input SP B, input Packets A, input Packets B, DES output, AOFF output, AOS_ON output, AOS_OFF output (OUT 2 of the circuit), and OUT 1.

BER measurements were performed for the input/output packets by using an error analyzer in the gated mode; the results are shown in Fig. 7. The corresponding eye diagrams in error-free operation are also shown in the figure. A power penalty of $0.6 \mathrm{~dB}, 1 \mathrm{~dB}, 1.3 \mathrm{~dB}$, and $1.7 \mathrm{~dB}$ with respect to the input packets (at a BER $=10^{-9}$ ) is observed for non-contending Packet A at OUT 1, contending Packet B at OUT 1 and deflected Packet B at OUT 2, and non-contending Packet B at OUT 1 , respectively.

The two last cases of interest, with more than two contending packets, are shown in Fig. 8(a) and (b). For the case of Fig. 8(a), one SP A interacts with two SPs $\mathrm{B}_{\mathrm{fs}}$ in the PM fiber, generating two BDGs in two different positions along the PM fiber. The probe pulse is thus initially partially reflected by the first grating (created by the interaction of SP A and the first $\mathrm{SP} \mathrm{B}_{\mathrm{fs}}$ entering in the PM fiber), producing a contention-detecting pulse signaling the advanced contending Packet $\mathrm{B}$. The transmitted probe is then reflected by the second grating created further ahead in the fiber (produced by the interaction of SP A and the second $\mathrm{SP} \mathrm{B}_{\mathrm{fs}}$ entering in the PM fiber). A second contention-detecting pulse signaling the delayed contending Packet B is generated.
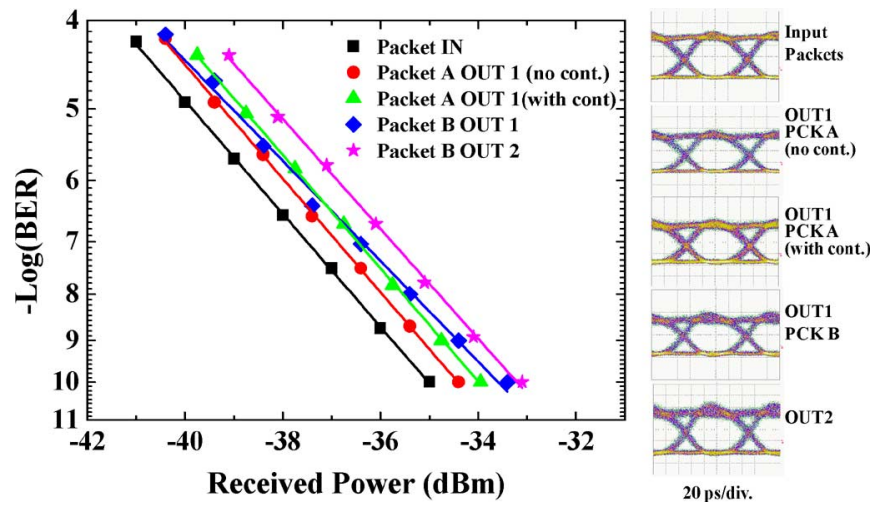

Fig. 7. BER curves. Insets: input/output eye diagrams.
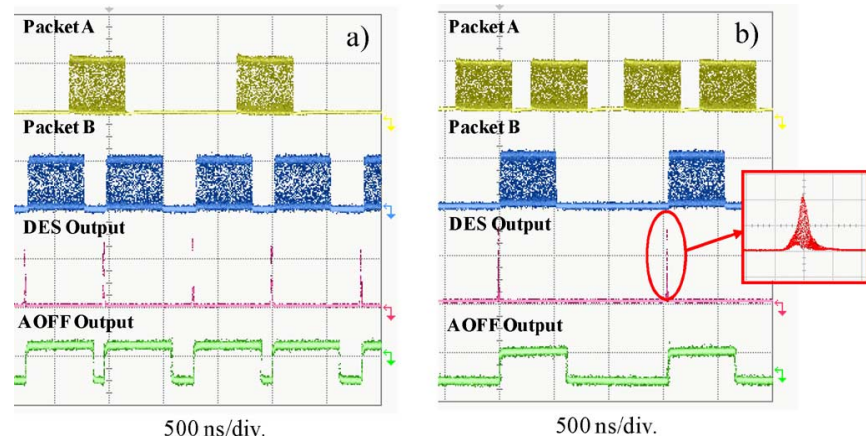

Fig. 8. Oscilloscope traces for (a): contention between two Packets B and one Packet A; b): contention between one packet B and two Packets A. From top to bottom: input Packets A, input Packets B, DES output, and AOFF output. The inset shows the details of the contention detecting pulse for case b).

Two switching signals are then properly produced by the AOFF in order to deflect the two contending LP packets, as illustrated in the figure. For the case of Fig. 8(b), two SP A and one SP $\mathrm{B}_{\mathrm{fs}}$ interact in the SBS-based ODL. The first SP A enters the fiber before $\mathrm{SP} \mathrm{B}_{\mathrm{fs}}$, and a second SP A is subsequently launched into the AOTDL before SP $\mathrm{B}_{\mathrm{fs}}$ exits the PM fiber. Two reflecting grating are thus created at two different locations along the $\mathrm{PM}$ fiber by the interaction of SP $\mathrm{B}_{\mathrm{fs}}$ with the two SPs A, causing two probe pulses to be reflected at different instants in different positions along the fiber. In particular, the first grating which is generated is created farther from the probe input side with respect to the grating which is generated at a later instant. The reflected probe signal that experiences the larger round-trip delay, is thus reflected before the probe signal which experiences less round-trip delay. Since the first reflected probe signal co-propagates with the SP $B_{\mathrm{fs}}$ (following SP $\mathrm{B}_{\mathrm{fs}}$ with a time delay of $\sim 2.5 \mathrm{~ns}$ ) it is easy to verify that it traverses the second grating at the same instant at which the second probe pulse is reflected from it. As a consequence, the two generated contention-detecting pulses exit the DES at the same instant. This can be verified also by considering that, since the two reflected probe pulses are signaling the same low-priority packet, the switching signal at the AOFF output must be the same for the two cases, implying that the two reflected probe pulses must leave the DES at the same instant. For this case, the output signal from the DES, which is the superposition of two reflected probe pulses, is shown in the inset of Fig. 8(b). Interference between the two 


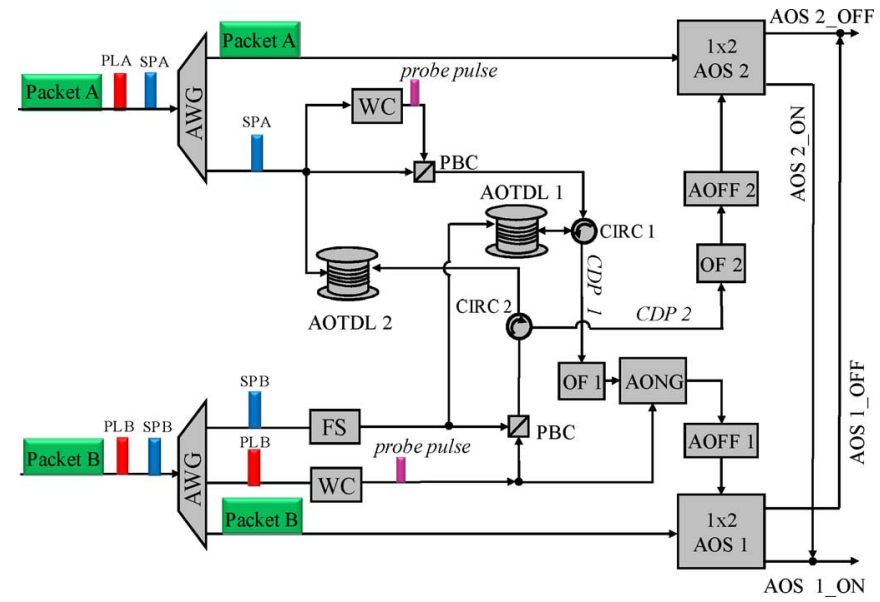

Fig. 9. Extension to priority swapping between incoming asynchronous packets. (PL: priority label; SP: synchronization pulse; WC: wavelength converter, FS: frequency shifter; PBC: polarization beam coupler; CIRC: circulator; CDP contention-detecting pulse, OF: optical filter; AOFF: all-optical flip-flop, AONG: all-optical NOT gate; AOS: all-optical switch.

overlapping pulses causes some degradation of the signal waveform; nevertheless, the noisy contention-detecting pulse can still correctly trigger the AOFF, as shown in Fig. 8(b). Finally, it should be noted that the exponential decaying feature of the DBG with a lifetime of $\sim 10 \mathrm{~ns}$ secures that the reflective grating is active only for the probe pulse associated with the SP A producing the Brillouin interaction, avoiding reflection of subsequent probe pulses for a typical packet length greater than few tens of ns.

\section{EXTENSION TO SYMMETRIC OPERATION}

In the experimental demonstration described in the previous sections, the packets' priority has been pre-assigned to a specific input port of the node, similarly to several previously reported contention resolution experiments (e.g., [15]-[17]). However, for increased node functionality and extended network flexibility, the possibility of handling priority regardless of the specific packet input port would be desirable. Dynamic priority assignment can be realized by taking decisions on the basis of a priority label, embedded in the header section of the incoming packets [18]. The extension of the scheme of Fig. 4 to such dynamic priority assignment operation is reported in Fig. 9. In the scheme, two SBS-based AOTDLs (AOTDL 1 and AOTDL 2 in the figure) are employed, each connected to an AOFF driving a $1 \times 2$ AOS. The input packets are comprised by a data payload, a SP field, and an additional priority label (PL), which can be either a logical "1" (signaling high priority request) or a logical " 0 ".

Similarly to what previously described, two parallel pump pulses are sent (from opposite sides) to the AOTDLs for the generation of the DBG which causes reflection of an orthogonally-polarized probe pulse in case of contention. In particular, for both the AOTDLs, the pump-pulses are provided by the SPs associated with the incoming packets (with proper frequency shift of SP B). For the case of AOTDL 1, the probe pulse is a wavelength-converted replica of SP A, and is sent from the same input port of AOTDL1 as SP A with a proper time delay.
On the other hand, for the case of AOTDL 2, the probe pulse is provided by wavelength-converting the priority label associated with Packet B, which enters the AOTDL 2 following the frequency-shifted SP B; the presence of the probe pulse in the AOTDL 2 thus depends on the value of the priority field in the incoming Packet B. When the priority label of Packet B is a logical "1", a reflected probe pulse is retrieved at AOTDL 2 output in case of contention and is exploited to drive AOFF 2 for space deflection of Packet A through the $1 \times 2$ AOS 2. The detailed operation of the scheme of Fig. 9 can be fully described with the aid of Table I, where the values of the contention-detecting pulses (CDPs) at the output of AOTDL 1 and AOTDL 2 and the corresponding $O N / O F F$ state of the AOFFs are specified for all the possible contention and priority cases. The first case, Case $I$ in Table I, is relative to the uninteresting situation in which no packet is present at both the node inputs. Similarly, Case II and Case III are relative to the trivial situations in which either solely Packet A or solely Packet B is present at IN 1 or IN 2, respectively. In these cases, regardless of the value of the priority labels, no contention-detecting pulse is generated, and both the AOSs remain in the OFF state, for which either Packet A or Packet B will be deviated toward the same output, the intended node output. In particular, in Case II, the probe pulse originated from SP A entering the AOTDL 1 trough CIRC 1 together with SP A, is not reflected and is lost at the output of the fiber span implementing the tunable delay line. Similarly, for Case III, either no probe pulse is delivered to AOTDL2 when the PL B is "0" for Packet B, or no reflected probe pulse exits AOTDL 2 if the priority field is " 1 " for Packet B. In both cases, both AOFF 1 and AOFF 2 remain idle causing either Packet B or Packet A to be deflected toward the intended node output through the AOS 1_ OFF and AOS 2_ OFF paths, respectively. Case IV of Table $\mathrm{I}$ is related to the more interesting situation in which a contention occurs; in particular, this case treats the circumstance in which both packets $\mathrm{A}$ and $\mathrm{B}$ are present at their respective inputs, and PL B is a logical " 0 ". Regardless of the value of PL $\mathrm{A}$, a DBG is created in AOTDL 1 by the interaction of SP A and frequency-shifted SP B, causing the reflection of the probe pulse following SP A.

A contention-detecting pulse (CDP 1) is retrieved after CIRC 1 , and after being isolated by OF 1 and polarizer (not shown in the figure for the sake of clarity), is delivered to AOFF 1 for the generation of a synchronized switching signal for AOS 1 deflecting Packet B toward the AOS 1_ON output (service output). At the opposite inputs of AOTDL 2 two pump pulses are present, but no probe signal is sent along the fiber, since PL $\mathrm{B}$ is set to " 0 ". No triggering signal is thus delivered to AOFF 2 and Packet A normally outputs the AOS 2_OFF path of AOS 2. Case IV, includes the situation in which both the priority fields of Packet A and Packet B are in the OFF state, which implies undefined priority. For this case of no specific request of priority, the circuit assumes that the packet entering from IN 1 has the highest priority. Last case, Case V in Table I, is relative to the situation in which both packets are present at the two node inputs and priority field of Packet B is in the high state (PL B set to "1"). As described before, regardless of the value of PL A, a pump and a probe pulse is present at one input of AOTDL 1, whereas a second frequency-shifted SP B pump pulse is present 
TABLE I

Operation OF THE CONTENTION RESOLUTION SCHEME OF Fig. 10

\begin{tabular}{|c|c|c|c|c|c|c|c|c|}
\hline & SP A & PL A & SP B & PL B & CDP 1 & CDP 2 & AOFF 1 & AOFF 2 \\
\hline $\begin{array}{c}\text { Case } \\
\text { I }\end{array}$ & 0 & 0 & 0 & 0 & 0 & 0 & OFF & OFF \\
\hline $\begin{array}{c}\text { Case } \\
\text { II }\end{array}$ & 1 & $0 / 1$ & 0 & 0 & 0 & 0 & OFF & OFF \\
\hline $\begin{array}{c}\text { Case } \\
\text { III }\end{array}$ & 0 & 0 & 1 & $1 / 0$ & 0 & 0 & OFF & OFF \\
\hline $\begin{array}{c}\text { Case } \\
\text { IV }\end{array}$ & 1 & $0 / 1$ & 1 & 0 & 0 & 1 & ON & OFF \\
\hline $\begin{array}{c}\text { Case } \\
\text { V }\end{array}$ & 1 & $0 / 1$ & 1 & 1 & 1 & 1 & OFF & ON \\
\hline
\end{tabular}

at its other input. A contention-detecting pulse (CDP 1) is thus present at CIRC 1 output and filtered by OF 1 . It is easy to verify (see for instance oscilloscope traces in Fig. 6) that CDP 1 is synchronous with SP B and, consequently, with PL B as well. The priority label PL B, which is now in the $O N$ state (PL B set to "1"), can then be conveniently used as a control signal in all-optical NOT gate (AONG) placed after OF 1, to suppress CDP 1 . This prevents the triggering of AOFF 1, thus leaving the AOS 1 in the $O F F$ state. As a consequence, the high-priority Packet B is routed toward the intended node output (AOS 2_OFF path). The all-optical NOT-gate can be simply implemented for instance by exploiting cross-gain compression in a SOA. In the AOTDL 2, a Bragg-like grating is also created by the interaction of SP A and the frequency-shifted SP B; a probe pulse, provided by PL $\mathrm{B}$, is also launched in the fiber following SP B. A contention-detecting pulse (CDP 2), synchronous with Packet $\mathrm{A}$, is then present at the output of CIRC 2 and delivered to AOFF 1 after being selected by OF 2 . This allows to synchronously deflect Packet A at AOS 2 toward the service output (AOS 2_ON path) and avoid collision with Packet B. This last case includes the unresolved situation in which both priority labels are in the $O N$ state for both the input packets, for which the scheme assigns priority to packets entering from IN 2. Even though the information of the priority field of Packet A is not required for the circuit operation, for full network transparency we have assumed all the Packets have the same structure, as depicted in Fig. 9.

\section{CONCLUSION}

A novel approach for all-optical resolution of contentions between asynchronous packets by means of an all-optical self-synchronizing mechanism in the routing node, suitable for fixed-length packets, has been proposed. The scheme exploits an all-optically tunable delay line based on dynamic Brillouin grating reflectors in PM fibers, capable to evaluate the time delay between synchronization pulses sent along with the input packets and correspondingly delay the output timing of a contention-detection pulse. The properly delayed contention-detecting pulse is then exploited to synchronize all-optical switches for deflection of low-priority packets in case of contention, by triggering an integrated all-optical flip-flop. The integrated all-optical flip-flop is based on a monolithic semiconductor ring laser, which exploits the inherent directional bistability among the two counter propagating modes supported by the laser cavity. We have experimentally demonstrated correct operation of the proposed scheme under different cases of contentions between asynchronous packets, showing moderate power penalties for the $10 \mathrm{~Gb} / \mathrm{s}$ payload data (below $1.7 \mathrm{~dB}$ ) without bit loss or packet fragmentation. The scheme operates entirely in the photonic domain, without exploitation of electrical control plane. By avoiding electrical signal processing, and exploiting semiconductor integrated optical switches and passive AOTDL, the scheme offers advantages in terms of reduced node latency. The scheme provides a practical system application of an all-optical continuously tunable variable delay line, showing the potentials of optically-controllable variable buffers as functional subsystem elements in optically packet-switched networks. Extension to symmetric operation allowing to handle packets' priorization has been also proposed and discussed.

\section{REFERENCES}

[1] Y.-K. Yeo, J. Yu, and G.-K. Chang, "A dynamically reconfigurable folded-path time delay buffer for optical packet switching," IEEE Photon. Technol. Lett., vol. 16, no. 11, pp. 2559-2561, Nov. 2004.

[2] Z. Wang, N. Chi, and S. Yu, "Time-slot assignment using optical buffer with a large variable delay range based on AVC crosspoint switch," $J$. Lightw. Technol., vol. 24, no. 8, pp. 2994-3001, Aug. 2006.

[3] C. J. Chang-Hasnian, P. C. Ku, J. Kim, and S. L. Chuang, "Variable optical buffer using slow light in semiconductor nanostructures," Proc. IEEE, vol. 11, pp. 1884-1897, 2003.

[4] M. L. Povinelli, S. G. Johnson, and J. D. Joannopoulos, "Slow-light, bandedge waveguides for tunable time delays," Opt. Exp., vol. 13, pp. 7145-7159, 2005.

[5] D. Dahan and G. Eisenstein, "Tunable all optical delay via slow and fast light propagation in a Raman assisted fiber optical parametric amplifiers: A route to all optical buffering," Opt. Exp., vol. 13, no. 16, pp. 6234-6249, Aug. 2005.

[6] J. E. Sharping, Y. Okawachi, and A. L. Gaeta, "Wide bandwidth slow light using a Raman fiber amplifier," Opt. Exp, vol. 13, no. 16, pp. 6092-6098, Aug. 2005.

[7] K. Y. Song, M. G. Herráez, and L. Thévenaz, "Observation of pulse delaying and advancement in optical fibers using stimulated Brillouin scattering," Opt. Exp., vol. 13, no. 1, pp. 82-88, Jan. 2005.

[8] Y. Okawachi, M. S. Bigelow, J. E. Sharping, Z. Zhu, A. Schweinsberg, D. J. Gauthier, R. W. Boyd, and A. L. Gaeta, "Tunable all-optical delays via Brillouin slow light in an optical fiber," Phys. Rev. Lett., vol. 19, no. 4, pp. 153902-1-4, Apr. 2005.

[9] J. E. Sharping, Y. Okawachi, J. van Howe, C. Xu, Y. Wang, A. E. Willner, and A. L. Gaeta, "All-optical, wavelength and bandwidth preserving, pulse delay based on parametric wavelength conversion and dispersion," Opt. Exp., vol. 13, no. 20, pp. 7872-7877, Oct. 2005.

[10] T. Kunihiro, T. Kanou, S. Oda, and A. Maruta, "Soliton self-frequency shift based slow light in optical fiber up to 1600 delay-to-pulse-width ratio," in Proc. ECOC 2007, Berlin, Germany, Sept. 16-20, 2007.

[11] N. Alic, E. Myslivets, S. Moro, B. P.-P. Kuo, R. M. Jopson, C. J. McKinstrie, and S. Radic, "Microsecond parametric optical delays," J. Lightw. Technol., vol. 28, no. 4, pp. 448-455, Feb. 2010.

[12] T. Schneider, K. Jamshidi, and S. Preussler, "Quasi-light storage: A method for the tunable storage of optical packets with a potential delaybandwidth product of several thousand bits," J. Lightw. Technol., vol. 28, no. 17, pp. 2586-2592, Sep. 2010.

[13] N. Primerov, S. Chin, K. Y. Song, and L. Thevenaz, "Ultra wide range tunable delay line using dynamic grating reflectors in optical fibers," in Proc. OFC 2010, San Diego, USA, Mar. 23-25, 2010, Paper OWF6.

[14] C. Porzi, S. Chin, A. Trita, F. Fresi, G. Berrettini, G. Mezosi, P. Ghelfi, G. Giuliani, L. Poti, M. Sorel, L. Thevenaz, and A. Bogoni, "All-optical self-synchronizing scheme for contention resolution in asynchronous optical packet switched networks using continuously tunable optical delay line," in Proc. OFC 2011, Los Angeles, CA, USA, Mar. 6-10, 2011, Paper JWA46.

[15] Y. Liu, M. T. Hill, R. Geldenhuys, N. Calabretta, H. de Waardt, G.-D. Kohe, and H. J. S. Dorren, "Demonstration of a variable optical delay for a recirculating buffer by using all-optical signal processing," IEEE Photon. Technol. Lett., vol. 16, pp. 1748-1750, Jul. 2004. 
[16] L. Stampoulidis, E. Kehayas, D. Apostopolopoulos, P. Bakopoulos, K. Vyrsokinos, and H. Avramopoulos, "On-the-fly all-optical contention resolution for NRZ and RZ data formats using packet envelope detection and integrated optical switches," IEEE Photon. Technol. Lett., vol. 19, pp. 538-540, Apr. 2007.

[17] C. Porzi, F. Fresi, L. Poti, A. Bogoni, M. Guina, L. Orsila, O. Okhotnikov, and N. Calabretta, "All-optical packet envelope detection using a slow semiconductor saturable absorber gate and a semiconductor optical amplifier," IEEE J. Sel. Topics Quantum Electron., vol. 14, no. 3, pp. 834-840, May/Jun. 2008.

[18] P. Bakopoulos, P. Zakynthinos, E. Kehayas, L. Stampoulidis, F. Fresi, C. Porzi, N. Calabretta, Ch. Kouloumentas, D. Petrantonakis, A. Maziotis, C. Stamatiadis, D. Apostolopoulos, M. Guina, D. Klonidis, L. Potì, E. Tangdiongga, A. Poustie, G. Maxwell, I. Tomkos, A. Bogoni, H. J. S. Dorren, and H. Avramopoulos, " $160 \mathrm{~Gb} / \mathrm{s}$ all-optical contention resolution with prioritization using integrated photonic components," in Proc. ECOC 2009, Vienna, Austria, Sep. 20-24, 2009, Paper 6.3.5.

[19] S. Rangarajan, Z. Hu, L. Rau, and D. J. Blumenthal, "All-optical contention resolution with wavelength conversion for asynchronous variable-length $40 \mathrm{~Gb} / \mathrm{s}$ optical packets," IEEE Photon. Technol. Lett., vol. 16, no. 2, pp. 689-691, Feb. 2004.

[20] D. Apostolopoulos, P. Zakynthinos, L. Stampoulidis, E. Kehayas, R. McDougall, R. Harmon, A. Poustie, G. Maxwell, R. Van Caenegem, D. Colle, M. Pickavet, E. Tangdiongga, H. J. S. Dorren, and H. Avramopoulos, "Contention resolution for burst-mode traffic using integrated SOA-MZI gate arrays and self-resetting optical flip-flops," IEEE Photon. Technol. Lett., vol. 20, no. 24, pp. 2024-2027, Dec. 2008.

[21] S. Yao, B. Mukherjee, S. J. B. Yoo, and S. Dixit, "A unified study of contention resolution schemes in optical packet-switched networks," $J$. Lightw. Technol., vol. 21, no. 3, pp. 672-683, Mar. 2003.
[22] S. J. B. Yoo, "Optical packet and burst switching technologies for the future photonic internet," J. Lightw. Technol., vol. 24, no. 12, pp. 4468-4492, Dec. 2006.

[23] H. Harai and M. Murata, "Optical fiber-delay-line buffer management in output-buffered photonic packet switch to support service differentiation," IEEE J. Sel. Areas Commun., vol. 24, no. 8, pp. 108-116, Aug. 2006.

[24] J. P. Mack, E. F. Burmeister, J. M. Garcia, H. N. Poulsen, B. Stamenic, G. Kurczveil, K. N. Nguyen, K. Hollar, J. E. Bowers, and D. J. Blumenthal, "Synchronous optical packet buffers," IEEE J. Sel. Topics Quantum Eletron., vol. 16, no. 5, pp. 1413-1421, Sep./Oct. 2010.

[25] T. Sakamoto, A. Okada, M. Hirayama, Y. Sakai, O. Moriwaki, I. Ogawa, R. Sato, K. Noguchi, and M. Matsuoka, "Optical packet synchronizer using wavelength and space switching," IEEE Photon. Technol. Lett., vol. 14, no. 9, pp. 1360-1362, Sep. 2002.

[26] F.-S. Choa, X. Zhao, X. Yu, J. Lin, J. P. Zhang, Y. Gu, G. Ru, G. Zhang, L. Li, H. Xiang, H. Hadimioglu, and H. J. Chao, "An optical packet switch based on WDM technologies," J. Lightw. Technol., vol. 23, no. 3, pp. 994-1014, Mar. 2005.

[27] Z. Wang, N. Chi, and S. Yu, "Time-slot assignment using optical buffer with a large variable delay range based on AVC crosspoint switch," $J$. Lightw. Technol., vol. 24, no. 8, pp. 2994-3001, Aug. 2006.

[28] K. J. Song, W. Zou, Z. He, and K. Hotate, "All-optical dynamic grating generation based on Brillouin scattering in polarization maintaining fiber," Opt. Lett., vol. 33, no. 9, pp. 926-928, May 2008.

[29] A. Trita, G. Mezosi, M. J. L. Vidal, M. Zanola, I. Cristiani, M. Sorel, P. Ghelfi, A. Bogoni, and G. Giuliani, " $10 \mathrm{~Gb} / \mathrm{s}$ operation of monolithic all-optical set-reset flip-flop based on semiconductor ring laser," in Proc. CLEO/QELS'10, San Jose, CA, USA, May 16-21, 2010, Paper CThN3. 